\title{
Fundamental Limits on Time Delay Estimation in Dispersed Spectrum Cognitive Radio Systems
}

\author{
Sinan Gezici, Hasari Celebi, H. Vincent Poor, and Huseyin Arslan
}

\begin{abstract}
In this paper, fundamental limits on time delay estimation are studied for cognitive radio systems, which facilitate opportunistic use of spectral resources. First, a generic CramerRao lower bound (CRLB) expression is obtained in the case of unknown channel coefficients and carrier-frequency offsets (CFOs) for cognitive radio systems with dispersed spectrum utilization. Then, various modulation schemes are considered, and the effects of unknown channel coefficients and CFOs on the accuracy of time delay estimation are quantified. Finally, numerical studies are performed in order to verify the theoretical analysis.
\end{abstract}

Index Terms-Dispersed spectrum utilization, time delay estimation, Cramer-Rao lower bound (CRLB), cognitive radio, carrier frequency offset (CFO).

\section{INTRODUCTION}

C OGNITIVE radio is a novel approach for intelligent wireless communications systems [1]-[3]. One of the prominent characteristics of cognitive radio is location awareness [4]-[6]. Cognitive radios with location awareness can support goal-driven autonomous location aware systems, such as location-based services (e.g., package tracking), location-assisted network optimization (e.g., dynamic spectrum management), location-assisted transceiver optimization (e.g., adaptive beamforming), and environment identification (e.g., propagation channel characterization) [4]. The main component of location awareness is to obtain accurate location information [7], [8]. Among various approaches for obtaining location information, time-based techniques are quite popular due to their accuracy and practicality [9], [10]. The main factors that affect the performance of time-based localization systems are accuracy of time delay estimation, the number of localization devices and their positions, and channel conditions, such as multipath and non-line-of-sight propagation [9], [11]-[13]. In this paper, theoretical limits on time delay estimation are studied for dispersed spectrum cognitive radio systems. Accurate time delay estimation is a crucial step in obtaining reliable location information in cognitive radio

Manuscript received January 24, 2008; revised June 28, 2008; accepted August 11, 2008. The associate editor coordinating the review of this letter and approving it for publication was S. Affes.

S. Gezici is with the Department of Electrical and Electronics Engineering, Bilkent University, Bilkent, Ankara TR-06800, Turkey (e-mail: gezici@ee.bilkent.edu.tr).

H. Celebi and H. Arslan are with the Department of Electrical Engineering, University of South Florida, Tampa, FL, 33620, USA (e-mail: hcelebi@mail.usf.edu; arslan@eng.usf.edu).

H. V. Poor is with the Department of Electrical Engineering, Princeton University, Princeton, NJ 08544, USA (e-mail: poor@princeton.edu.)

This work was supported in part by the European Commission in the framework of the FP7 Network of Excellence in Wireless COMmunications NEWCOM++ (contract no. 216715), and in part by the U. S. National Science Foundation under Grants ANI-03-38807 and CNS-06-25637.

Digital Object Identifier 10.1109/T-WC.2009.080104 systems. Therefore, investigation of theoretical limits on time delay estimation is of considerable importance.

Cognitive radio systems with location awareness can use Cramer-Rao lower bound (CRLB) information at the transmitter side to optimize the transmission parameters for achieving goal-driven accuracy requirements. In [7], the CRLB for time delay estimation in multipath channels is derived in the presence of unknown path delays and coefficients. This study is extended in [14] by deriving the frequency-domain CRLB in dynamic spectrum access systems under the assumption that path delays, distance-dependent coefficients, phases and frequency-dependent coefficients are unknown. Furthermore, the effects of bandwidth, carrier frequency, and path frequency dependence on the CRLB in dynamic spectrum access systems are investigated via computer simulations. The studies in [7] and [14] are based on the fact that the available bandwidth is in the form of a single piece (i.e., the whole bandwidth). Since the available bandwidths in dynamic spectrum access systems are commonly dispersed [15], a single cognitive radio user can transmit and receive over multiple dispersed bands. Such systems are referred to as dispersed spectrum utilization systems and are developed in the context of time delay estimation in this study.

In this paper, fundamental limits on time delay estimation are studied in dispersed spectrum utilization systems, where the available dispersed bandwidths are assumed to be narrow. The limits are obtained in terms of the CRLBs and the effects of both unknown channel coefficients and carrier frequency offsets (CFOs) are taken into account. In this way, the effects of dispersed spectrum utilization on the accuracy of time delay estimation are quantified explicitly. After deriving a generic expression, specific CRLB formulas are obtained for various modulation schemes. It is shown for linear modulation schemes that the same fundamental limits can be achieved for the cases of known and unknown CFOs. In addition, it is proven that the effects of unknown channel coefficients are mitigated as well for linear modulation formats with constant envelopes. Finally, numerical results are presented to verify the theoretical analysis.

\section{Signal Model}

Consider a cognitive radio system that occupies $K$ different frequency bands as shown in Fig. 1. The cognitive transmitter sends a signal occupying all the $K$ bands, and the receiver wishes to calculate the time delay of the incoming signal. One way to implement such a system is to consider the received signal as an orthogonal frequency division multiplexing (OFDM) signal with zero coefficients at the sub-carriers corresponding to the unused bands [16]-[18]. Then, the signal 


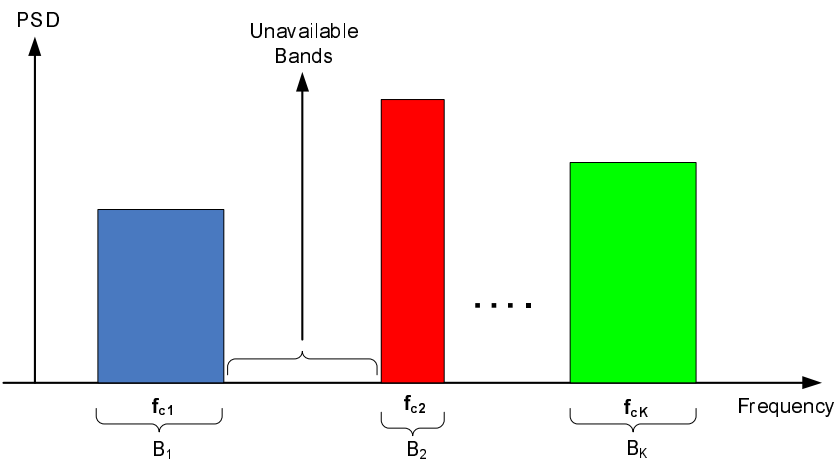

Fig. 1. Illustration of dispersed spectrum utilization in cognitive radio systems.

can be processed as in a conventional OFDM receiver. However, the available spectrum can be very dispersed in some cases, which requires processing of very large bandwidths if the signal is considered as a single OFDM signal. In such cases, it can be more practical to process the signal in multiple branches, as shown in Fig. 2. Each branch considers one available band, and down-converts the signal according to the center frequency of that band. In this way, signals with narrower bandwidths can be processed at each branch. Then, the question is to determine the accuracy of time delay estimation for such a receiver structure, considering practical issues such as CFO due to the use of multiple down-conversion units.

The baseband representation of the received signal in the $i$ th branch can be expressed as

$$
r_{i}(t)=\alpha_{i} \mathrm{e}^{-j \omega_{i} t} s_{i}(t-\tau)+n_{i}(t)
$$

for $i=1, \ldots, K$, where $\alpha_{i}=a_{i} \mathrm{e}^{j \phi_{i}}$ and $\omega_{i}$ represent, respectively, the channel coefficient and the $\mathrm{CFO}$ for the signal in the $i$ th branch, $s_{i}(t)$ is the baseband representation of the transmitted signal corresponding to the $i$ th band, $\tau$ is the time delay, and $n_{i}(t)$ is complex Gaussian noise with independent and white components, each having spectral density $\sigma_{i}^{2}$.

For the signal model in (1), it is assumed that the signal at each branch can be modeled as a narrowband signal. Therefore, a single complex channel coefficient is used to represent the fading of each signal.

Note that, in addition to the cognitive radio framework, the signal model in (1) can also represent a multiple-input multiple-output (MIMO) system, in which transmitter $i$ sends $s_{i}(t)$ to receiver $i$, in the presence of synchronization among the transmissions, or when the receiver knows the relative delays between transmissions.

\section{CRLB CALCUlations}

Let $\boldsymbol{\theta}=\left[\begin{array}{llllll}\tau & a_{1} \cdots a_{K} & \phi_{1} \cdots \phi_{K} & \omega_{1} \cdots \omega_{K}\end{array}\right]$ represent the vector of unknown signal parameters. If the signals in (1) are observed over the interval $[0, T]$, the log-likelihood function

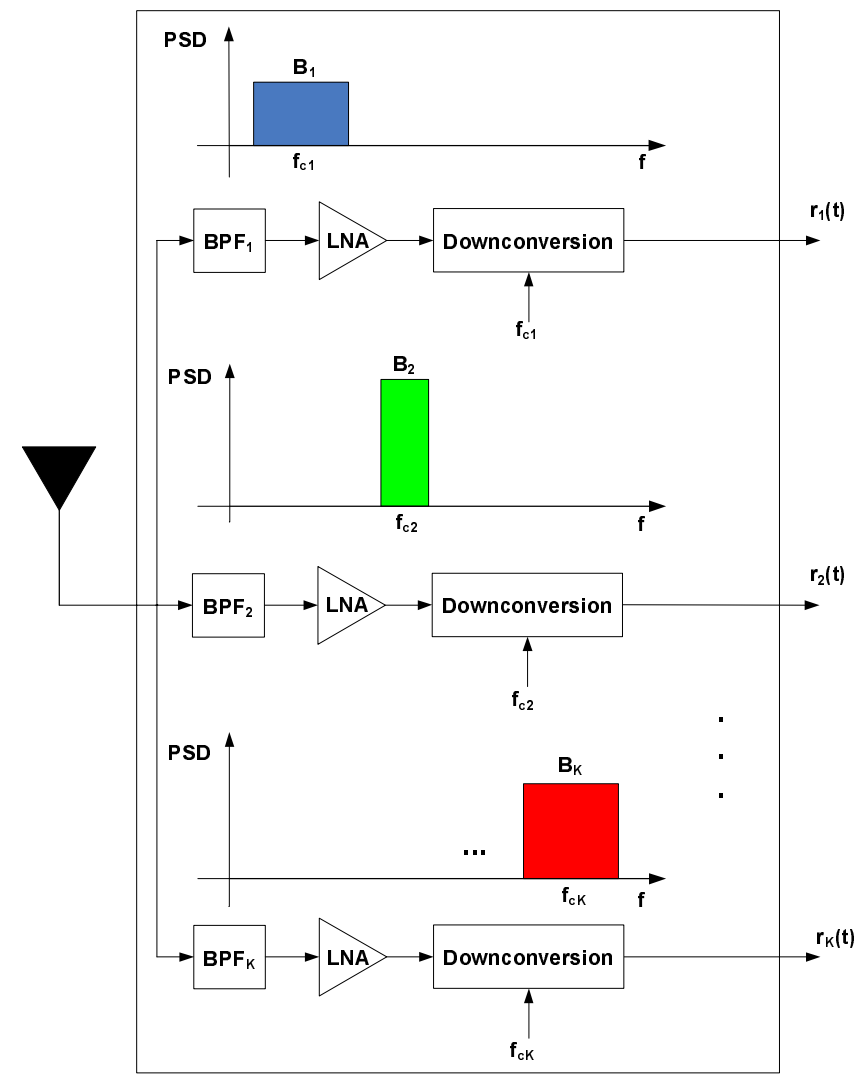

Fig. 2. Block diagram of a cognitive radio receiver.

for $\boldsymbol{\theta}$ is given by ${ }^{1}$ [19]

$$
\Lambda(\boldsymbol{\theta})=c-\sum_{i=1}^{K} \frac{1}{2 \sigma_{i}^{2}} \int_{0}^{T}\left|r_{i}(t)-\alpha_{i} \mathrm{e}^{j \omega_{i} t} s_{i}(t-\tau)\right|^{2} \mathrm{~d} t,
$$

where $c$ is a constant that is independent of $\boldsymbol{\theta}$. Then, the maximum likelihood (ML) estimate for $\boldsymbol{\theta}$ can be obtained from (2) as ${ }^{2}$

$$
\begin{aligned}
\hat{\boldsymbol{\theta}}_{\mathrm{ML}}=\arg \max _{\boldsymbol{\theta}}\{ & \sum_{i=1}^{K} \frac{1}{\sigma_{i}^{2}} \int_{0}^{T} \mathcal{R}\left\{\alpha_{i}^{*} \mathrm{e}^{-j \omega_{i} t} r_{i}(t) s_{i}^{*}(t-\tau)\right\} \mathrm{d} t \\
& \left.-\sum_{i=1}^{K} \frac{E_{i}\left|\alpha_{i}\right|^{2}}{2 \sigma_{i}^{2}}\right\}
\end{aligned}
$$

where $E_{i}=\int_{0}^{T}\left|s_{i}(t-\tau)\right|^{2} \mathrm{~d} t$ is the signal energy ${ }^{3}$.

From (2), the Fisher information matrix (FIM) [19] can be obtained, after some manipulation, as

$$
\mathbf{I}=\left[\begin{array}{cccc}
\mathbf{I}_{\tau \tau} & \mathbf{I}_{\tau a} & \mathbf{I}_{\tau \phi} & \mathbf{I}_{\tau \omega} \\
\mathbf{I}_{\tau a}^{T} & \mathbf{I}_{a a} & \mathbf{0} & \mathbf{0} \\
\mathbf{I}_{\tau \phi}^{T} & \mathbf{0} & \mathbf{I}_{\phi \phi} & \mathbf{I}_{\phi \omega} \\
\mathbf{I}_{\tau \omega}^{T} & \mathbf{0} & \mathbf{I}_{\phi \omega}^{T} & \mathbf{I}_{\omega \omega}
\end{array}\right],
$$

${ }^{1}$ It is assumed that the unknown parameters are constant during the observation interval.

${ }^{2}$ For a complex number $z, \mathcal{R}\{z\}$ and $\mathcal{I}\{z\}$ represent its real and imaginary parts, respectively.

${ }^{3}$ Although $E_{i}$ is a function of $\tau$ in general, this dependence is not shown explicitly for convenience. The same convention is also employed for the expressions in (13)-(17). 
with

$$
\begin{aligned}
\mathrm{I}_{\tau \tau} & =\sum_{i=1}^{K} \gamma_{i} \tilde{E}_{i}, \\
\mathbf{I}_{a a} & =\operatorname{diag}\left\{\frac{E_{1}}{\sigma_{1}^{2}}, \ldots, \frac{E_{K}}{\sigma_{N}^{2}}\right\}, \\
\mathbf{I}_{\phi \phi} & =\operatorname{diag}\left\{E_{1} \gamma_{1}, \ldots, E_{K} \gamma_{K}\right\}, \\
\mathbf{I}_{\omega \omega} & =\operatorname{diag}\left\{F_{1} \gamma_{1}, \ldots, F_{K} \gamma_{K}\right\}, \\
\mathbf{I}_{\tau a} & =-\left[\hat{E}_{1}^{\mathrm{R}} \frac{\left|\alpha_{1}\right|}{\sigma_{1}^{2}} \ldots \hat{E}_{K}^{\mathrm{R}} \frac{\left|\alpha_{K}\right|}{\sigma_{K}^{2}}\right], \\
\mathbf{I}_{\tau \phi} & =-\left[\hat{E}_{1}^{\mathrm{I}} \gamma_{1} \cdots \hat{E}_{K}^{\mathrm{I}} \gamma_{K}\right], \\
\mathbf{I}_{\tau \omega} & =-\left[G_{1} \gamma_{1} \cdots G_{K} \gamma_{K}\right], \\
\mathbf{I}_{\phi \omega} & =\operatorname{diag}\left\{\hat{F}_{1} \gamma_{1}, \ldots, \hat{F}_{K} \gamma_{K}\right\},
\end{aligned}
$$

where $\gamma_{i}=\left|\alpha_{i}\right|^{2} / \sigma_{i}^{2}, \operatorname{diag}\left\{x_{1}, \ldots, x_{K}\right\}$ represents an $K \times K$ diagonal matrix with its $i$ th diagonal being equal to $x_{i}, \tilde{E}_{i}$ is the energy of the first derivative of $s_{i}(t)$; i.e., $\tilde{E}_{i}=$ $\int_{0}^{T}\left|s_{i}^{\prime}(t-\tau)\right|^{2} \mathrm{~d} t$, and $\hat{E}_{i}^{\mathrm{R}}, \hat{E}_{i}^{\mathrm{I}}, F_{i}, \hat{F}_{i}$ and $G_{i}$ are given, respectively, by

$$
\begin{aligned}
\hat{E}_{i}^{\mathrm{R}} & =\int_{0}^{T} \mathcal{R}\left\{s_{i}^{\prime}(t-\tau) s_{i}^{*}(t-\tau)\right\} \mathrm{d} t, \\
\hat{E}_{i}^{\mathrm{I}} & =\int_{0}^{T} \mathcal{I}\left\{s_{i}^{\prime}(t-\tau) s_{i}^{*}(t-\tau)\right\} \mathrm{d} t, \\
F_{i} & =\int_{0}^{T} t^{2}\left|s_{i}(t-\tau)\right|^{2} \mathrm{~d} t, \\
\hat{F}_{i} & =\int_{0}^{T} t\left|s_{i}(t-\tau)\right|^{2} \mathrm{~d} t, \\
G_{i} & =\int_{0}^{T} t \mathcal{I}\left\{s_{i}^{*}(t-\tau) s_{i}^{\prime}(t-\tau)\right\} \mathrm{d} t .
\end{aligned}
$$

The CRLB for unbiased time delay estimators can be obtained from the element in the first row and first column of the inverse of the FIM in (4), i.e., $\left[\mathbf{I}^{-1}\right]_{11}$. Based on the formulas for block matrix inversion, the CRLB can be obtained as (Appendix A)

$$
\mathrm{CRLB}_{1}=\frac{1}{\sum_{i=1}^{K} \gamma_{i}\left(\tilde{E}_{i}-\left(\hat{E}_{i}^{\mathrm{R}}\right)^{2} / E_{i}\right)-\xi},
$$

where

$$
\xi=\sum_{i=1}^{K} \gamma_{i} \frac{\left(\hat{E}_{i}^{\mathrm{I}}\right)^{2} F_{i}+E_{i} G_{i}^{2}-2 \hat{E}_{i}^{\mathrm{I}} G_{i} \hat{F}_{i}}{E_{i} F_{i}-\hat{F}_{i}^{2}} .
$$

In order to investigate the effects of unknown CFOs, the CRLB for time delay estimation can be obtained for known CFOs. In that case, the unknown parameter vector reduces to $\tilde{\boldsymbol{\theta}}=\left[\begin{array}{lllll}\tau & a_{1} & \cdots & a_{K} & \phi_{1} \\ \cdots & \phi_{K}\end{array}\right]$, and the CRLB can be obtained, similarly to the previous derivations, as

$$
\mathrm{CRLB}_{2}=\frac{1}{\sum_{i=1}^{K} \gamma_{i}\left(\tilde{E}_{i}-\hat{E}_{i}^{2} / E_{i}\right)},
$$

where

$$
\hat{E}_{i}=\left|\int_{-\infty}^{\infty} s_{i}^{\prime}(t-\tau) s_{i}^{*}(t-\tau) \mathrm{d} t\right|=\sqrt{\left(\hat{E}_{i}^{\mathrm{R}}\right)^{2}+\left(\hat{E}_{i}^{\mathrm{I}}\right)^{2}} .
$$

Note that $\mathrm{CRLB}_{2}$ in (20) is smaller than or equal to $\mathrm{CRLB}_{1}$ in (18) in general, since more unknown parameters exist for the latter.

If both the channel coefficients and the CFOs are known, the unknown parameter vector reduces to $\tau$. Then, the CRLB can be obtained from (5) as

$$
\mathrm{CRLB}_{3}=\frac{1}{\sum_{i=1}^{K} \gamma_{i} \tilde{E}_{i}} .
$$

\section{Special Cases}

Although the CRLB can be obtained from (13)-(19) in general, its evaluation for specific signal structures can provide more intuition related to the effects of CFOs.

Let the baseband signal $s_{i}(t)$ in (1) consist of a sequence of modulated pulses as follows:

$$
s_{i}(t)=\sum_{l} d_{i, l}(t) p_{i}\left(t-l T_{i}\right),
$$

for $i=1, \ldots, K$, where $d_{i, l}(t)$ denotes the complex data ${ }^{4}$ for the $l$ th symbol of signal $i$, and $p_{i}(t)$ represents a pulse with duration $T_{i}$, i.e., $p_{i}(t)=0$ for $t \notin\left[0, T_{i}\right]$. To simplify notation, it is assumed that the observation interval $T$ can be expressed as $T=N_{i} T_{i}$ for an integer $N_{i}$ for $i=1, \ldots, K$.

Proposition 1: For any linear modulation of the form $s_{i}(t)=\sum_{l} d_{i, l} p_{i}\left(t-l T_{i}\right)$, the CRLBs in (18) and (20) are equal, and are given by

$$
\mathrm{CRLB}_{1}=\mathrm{CRLB}_{2}=\frac{1}{\sum_{i=1}^{K} \gamma_{i}\left(\tilde{E}_{i}-\left(\hat{E}_{i}^{\mathrm{R}}\right)^{2} / E_{i}\right)} .
$$

Proof: Let $s_{i}(t)=\sum_{l} d_{i, l}(t) p_{i}\left(t-l T_{i}\right)$ with $d_{i, l}(t)=d_{i, l}$ $\forall t$, as stated in the proposition. Then, $s_{i}^{\prime}(t) s_{i}^{*}(t)$ can be calculated from (23) as

$$
s_{i}^{\prime}(t) s_{i}^{*}(t)=\sum_{l}\left|d_{i, l}\right|^{2} p_{i}\left(t-l T_{i}\right) p_{i}^{\prime}\left(t-l T_{i}\right),
$$

which is always real. Since $s_{i}^{\prime}(t) s_{i}^{*}(t)$ is a real quantity, it can be shown that $\hat{E}_{i}^{\mathrm{I}}$ in (14) and $G_{i}$ in (17) are equal to zero. Therefore, it can be observed from (19) and (21) that CRLB 1 in (18) and $\mathrm{CRLB}_{2}$ in (20) reduce to the expression stated in the proposition.

Proposition 1 states that for most modulation types, such as pulse amplitude modulation (PAM), phase shift keying (PSK) and quadrature amplitude modulation (QAM) [20], the CRLB of time delay estimation for the case of unknown CFOs is the same as that for the case of known $\mathrm{CFOs}^{5}$. For non-linear modulation types, the statement in the proposition cannot be employed. As an example, for frequency shift keying (FSK) modulation with $d_{i, l}(t)=\exp \left\{j 2 \pi \tilde{d}_{i, l} \Delta_{i} t\right\}$, where $\tilde{d}_{i, l}$ is the modulation data and $\Delta_{i}$ is the amount of frequency shift, $s_{i}^{\prime}(t) s_{i}^{*}(t)$ is no longer a real quantity; hence, $\hat{E}_{i}^{\mathrm{I}}$ in (14) and $G_{i}$ in (17) are non-zero in general.

Proposition 2: For linear modulation of the form $s_{i}(t)=$ $\sum_{l} d_{i, l} p_{i}\left(t-l T_{i}\right)$, with $\left|d_{i, l}\right|=\left|d_{i}\right| \forall l$ and $p_{i}(t)$ satisfying

${ }^{4}$ Since a data-aided time delay estimation scenario is considered, the data symbols are assumed to be known.

${ }^{5}$ Unknown channel coefficients are considered for both cases. 
$p_{i}(0)=p_{i}\left(T_{i}\right)$ for $i=1, \ldots, K$, the CRLBs in (18), (20) and (22) are given by

$$
\mathrm{CRLB}_{1}=\mathrm{CRLB}_{2}=\mathrm{CRLB}_{3}=\frac{1}{4 \pi^{2} \sum_{i=1}^{K} \mathrm{SNR}_{i} \beta_{i}^{2}},
$$

where $\mathrm{SNR}_{i}=N_{i}\left|d_{i}\right|^{2} \frac{\left|\alpha_{i}\right|^{2} E_{p_{i}}}{\sigma_{i}^{2}}$ with $E_{p_{i}}=\int_{-\infty}^{\infty} p_{i}^{2}(t) \mathrm{d} t$, and $\beta_{i}$ is the effective bandwidth of $p_{i}(t)$, given by

$$
\beta_{i}^{2}=\frac{1}{E_{p_{i}}} \int_{-\infty}^{\infty} f^{2}\left|P_{i}(f)\right|^{2} \mathrm{~d} f,
$$

with $P_{i}(f)$ denoting the Fourier transform of $p_{i}(t)$.

Proof: Since $\left|d_{i, l}\right|=\left|d_{i}\right| \forall l, \hat{E}_{i}^{\mathrm{R}}$ in (13) can be obtained from (25) as

$$
\begin{aligned}
\hat{E}_{i}^{\mathrm{R}} & =\left|d_{i}\right|^{2} \sum_{l} \int_{0}^{T} p_{i}\left(t-\tau-l T_{i}\right) p_{i}^{\prime}\left(t-\tau-l T_{i}\right) \mathrm{d} t \\
& =N_{i}\left|d_{i}\right|^{2} \int_{-\infty}^{\infty} p_{i}(t) p_{i}^{\prime}(t) \mathrm{d} t=0,
\end{aligned}
$$

for $i=1, \ldots, K$. Note that the second equality is obtained from the fact that $T=N_{i} T_{i}$, and the final equality is due to the facts that $p_{i}(t)$ is non-zero only over $t \in\left[0, T_{i}\right]$ and satisfies $p_{i}(0)=p_{i}\left(T_{i}\right)$.

Since $\hat{E}_{i}^{\mathrm{R}}=0$ for $i=1, \ldots, K$, the CRLB expression in (24) becomes

$$
\mathrm{CRLB}_{1}=\mathrm{CRLB}_{2}=\frac{1}{\sum_{i=1}^{K} \gamma_{i} \tilde{E}_{i}},
$$

which is the same as the CRLB in (22) for the case of known CFOs and channel coefficients.

In addition, $\tilde{E}_{i}$ can be calculated, from (23) and the fact that $\left|d_{i, l}\right|=\left|d_{i}\right| \forall l$, as

$$
\begin{aligned}
& \tilde{E}_{i}=\int_{0}^{T}\left|s_{i}^{\prime}(t-\tau)\right|^{2} \mathrm{~d} t \\
& =\left|d_{i}\right|^{2} \sum_{l} \int_{0}^{T}\left(p_{i}^{\prime}\left(t-\tau-l T_{i}\right)\right)^{2} \mathrm{~d} t=N_{i}\left|d_{i}\right|^{2} \tilde{E}_{p_{i}},
\end{aligned}
$$

where $\tilde{E}_{p_{i}}=\int_{-\infty}^{\infty}\left(p_{i}^{\prime}(t)\right)^{2} \mathrm{~d} t$.

From Parseval's relation, $\tilde{E}_{p_{i}}$ can be expressed as $4 \pi^{2} \beta_{i}^{2} E_{p_{i}}$, where $E_{p_{i}}$ is the energy of $p_{i}(t)$ and $\beta_{i}$ is the effective bandwidth of $p_{i}(t)$ [19]. Then, it is observed from (31) that (30) is equal to (26) for $\mathrm{SNR}_{i}=N_{i}\left|d_{i}\right|^{2} \gamma_{i} E_{p_{i}}$ and $\gamma_{i}=\left|\alpha_{i}\right|^{2} / \sigma_{i}^{2}$.

From Proposition 2, it is observed that for linear modulation formats with constant envelope, such as PSK, the CRLB for time delay estimation is the same whether the CFOs and/or channel coefficients are known or unknown for pulses satisfying $p(0)=p(T)$, which is usually the case in practice. This implies that in such cases, the ML estimators in the absence of any information on CFOs and channel coefficients can asymptotically achieve the same CRLB as the ones in presence of CFO and/or channel coefficient information [19].

\section{NumericAl RESUlTS}

In this section, numerical studies are described that illustrate the CRLBs discussed in Sections III and IV. It is assumed that all the $K$ bands in the system have the same bandwidth and the same pulse is employed for all of them; i.e., $p_{i}(t)=p(t)$ for $i=1, \ldots, K$ (c.f. (23)). For the pulse shape, the following Gaussian doublet is employed ${ }^{6}$

$$
p(t)=A\left(1-\frac{4 \pi(t-1.25 \zeta)^{2}}{\zeta^{2}}\right) e^{-2 \pi(t-1.25 \zeta)^{2} / \zeta^{2}},
$$

where $A$ and $\zeta$ are parameters that are used to adjust the pulse energy and the pulse width, respectively. In the following, $\zeta=1 \mu \mathrm{s}$ is employed, for which the pulse width becomes approximately $2.5 \mu \mathrm{s}$, and $A$ is selected in order to generate pulses with unit energy. In addition, it is assumed that the spectral density of the noise is the same for all the $K$ branches; i.e., $\sigma_{i}=\sigma$ for $i=1, \ldots, K$, and the system SNR is defined as the sum of the SNRs in the various branches.

In Fig. 3, the CRLB expressions in (18), (20) and (22), labeled as $\mathrm{CRLB}_{1}, \mathrm{CRLB}_{2}$ and $\mathrm{CRLB}_{3}$, respectively, are plotted versus SNR for three different modulation types, namely $16 \mathrm{FSK}^{7}, 16 \mathrm{QAM}$, and $16 \mathrm{PSK}$. For all cases, it is assumed that the same modulation sequence is used at different branches; i.e., $d_{i, l}(t)=d_{l}(t)$ for $i=1, \ldots, K$, and that the channel amplitudes are normalized to unity; i.e., $\left|\alpha_{i}\right|=1$ for $i=1, \ldots, K$. In addition, the modulation sequence for each modulation type is scaled appropriately so that the sequences have the same energy in all cases. Also, there are $K=3$ branches in the system, and $N=2$ symbols are received at each branch. From the figure, it is first observed that for all the modulations, $\mathrm{CRLB}_{3} \leq \mathrm{CRLB}_{2} \leq \mathrm{CRLB}_{1}$ is satisfied, since $\mathrm{CRLB}_{1}$ corresponds to the case of unknown delay, channel coefficients and CFOs, $\mathrm{CRLB}_{2}$ corresponds to the case of unknown delay and channel coefficients, and CRLB $_{3}$ corresponds to the case of unknown delay only. In other words, for the cases with fewer unknown parameters, lower CRLBs are observed. For the 16FSK modulation, all three bounds are distinct, which is possible because 16FSK is a non-linear modulation and the result of Proposition 1 does not apply in this case. For the $16 \mathrm{QAM}$ case, $\mathrm{CRLB}_{1}=\mathrm{CRLB}_{2}$ as expected from Proposition 1. However, $\mathrm{CRLB}_{3}$ is lower than both, which is due to the fact that 16QAM is not a constant envelope modulation, for which the equivalence of all the CRLBs is not guaranteed as can be deduced from Proposition 2. Finally, the results for the 16PSK case show that all the three bounds are the same, which verifies the statement in Proposition 2.

In Fig. 4, $N=16$ symbols are considered and the rest of the system parameters are the same as in the previous case. It is observed that the results are similar to those in Fig. 3, except that the gap between the CRLBs is decreased, and lower CRLBs are attained when more symbols are employed. Also, the reduction of the gaps between $\mathrm{CRLB}_{1}, \mathrm{CRLB}_{2}$ and $\mathrm{CRLB}_{3}$ implies that using a larger number of observation symbols can mitigate the effects of unknown CFOs and/or channel coefficients.

Next, the CRLBs for different numbers of branches, which represent the numbers of available dispersed bands in the spectrum, are investigated for various SNR values. The parameters are the same as in the previous scenario, except that only $16 \mathrm{PSK}$ is considered here for simplicity, and the

\footnotetext{
${ }^{6}$ Although the pulse in (32) has infinite support, it can be assumed to have finite support of $[0,2.5 \zeta]$ in practice.

${ }^{7}$ The amount of frequency shift is selected as $190 \mathrm{kHz}$ for the FSK modulation.
} 


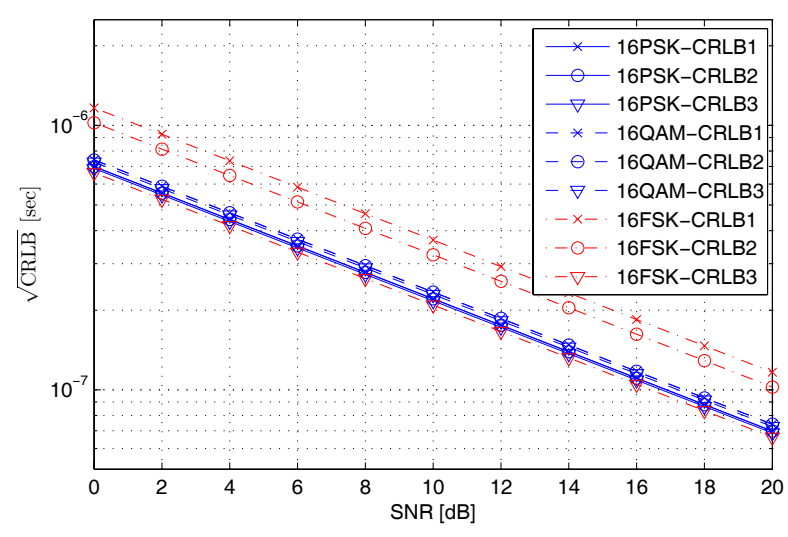

Fig. 3. $\sqrt{\mathrm{CRLB}}$ versus SNR for $K=3$ and $N=2$.

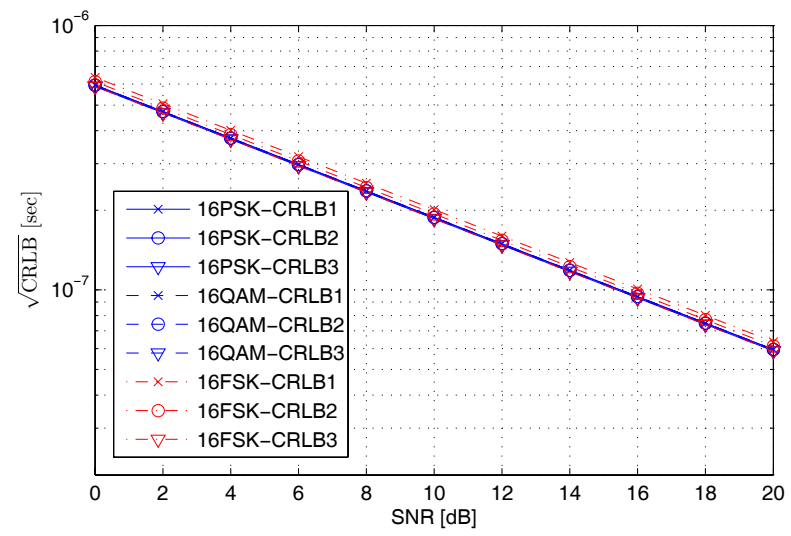

Fig. 4. $\sqrt{\mathrm{CRLB}}$ versus SNR for $K=3$ and $N=16$.

CRLB is evaluated for $K=1,2,3,4,5$ branches and for $\mathrm{SNR}=5,10,15 \mathrm{~dB}$. It is observed from Fig. 5 that the CRLBs decrease as the SNR increases but they stay the same for different numbers of branches. This is due mainly to the fact that the SNR is defined as the sum of the SNRs in the different branches; hence, the SNR per branch is reduced as more branches are considered (c.f. (26)). However, in some cases, as more bands become available for the cognitive radio system, higher SNRs can be achieved. For example, in the presence of a limit on the average power spectral density of the transmitted signal (e.g., the FCC regulations on ultrawideband systems [10]), the transmit power (hence, SNR) can be increased as more bands become available. For Fig. 6, the SNR is defined per branch and the total SNR is increased as $K$ increases. In this case, it is observed that as more bands become available, lower CRLBs can be achieved.

\section{CONCLUding REMARKS}

In this paper, CRLBs for time delay estimation have been obtained for dispersed spectrum utilization systems in the presence of unknown channel coefficients and CFOs. In addition, various modulation schemes have been considered and the effects of unknown channel coefficients and CFOs have been investigated.

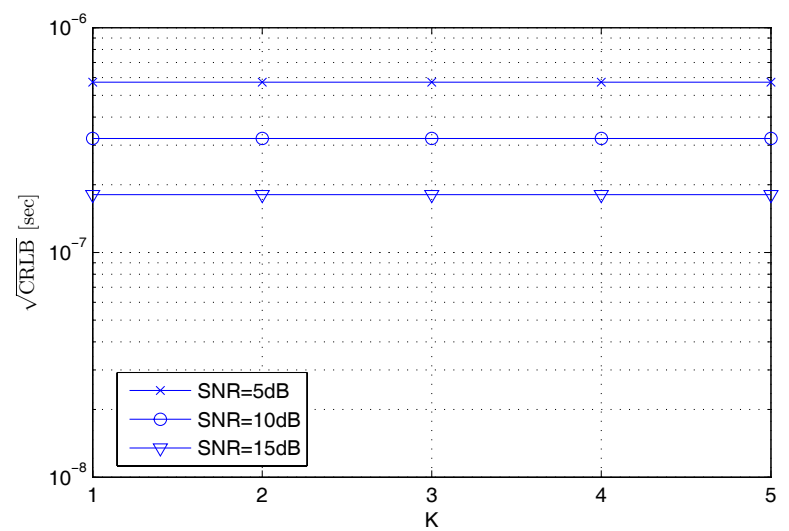

Fig. 5. $\sqrt{\mathrm{CRLB}}$ versus $K$ for $N=16$ and 16PSK modulation when the SNR is defined as the sum of the SNRs at different branches.

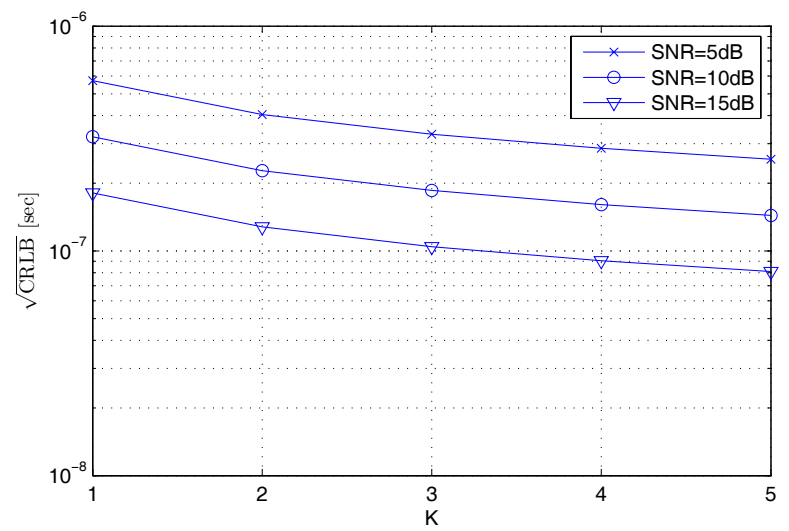

Fig. 6. $\sqrt{\mathrm{CRLB}}$ versus $K$ for $N=16$ and 16PSK modulation when the SNR is defined per branch.

Cognitive radio systems can employ CRLB information at the transmitter side for achieving range accuracy adaptation [7]. The results of this study indicate that range accuracy can be adapted by selecting an appropriate number $(K)$ of dispersed bands in the spectrum, modulation type, number of observation symbols, and/or SNR levels. This can be achieved by using the adaptive waveform generation feature of cognitive radios. Moreover, in order for a cognitive positioning system to achieve a given accuracy, it needs to take into account all impairments in the transmitter-channel-receiver chain. The results in this study (e.g., Fig. 4) suggest that impairments such as CFOs can be mitigated by selecting an appropriate modulation type, the number of observation symbols, and the SNR level.

\section{APPENDIX}

A. Derivation of (18) and (19):

Let $\mathbf{A}=\left[\begin{array}{cc}\mathrm{I}_{\tau \tau} & \mathbf{I}_{\tau a} \\ \mathbf{I}_{\tau a}^{T} & \mathbf{I}_{a a}\end{array}\right], \quad \mathbf{B}=\left[\begin{array}{cc}\mathbf{I}_{\tau \phi} & \mathbf{I}_{\tau \omega} \\ \mathbf{0} & \mathbf{0}\end{array}\right]$ and $\mathbf{D}=$ $\left[\begin{array}{ll}\mathbf{I}_{\phi \phi} & \mathbf{I}_{\phi \omega} \\ \mathbf{I}_{\phi \omega}^{T} & \mathbf{I}_{\omega \omega}\end{array}\right]$. Then, $\mathbf{I}$ can be expressed as $\mathbf{I}=\left[\begin{array}{cc}\mathbf{A} & \mathbf{B} \\ \mathbf{B}^{T} & \mathbf{D}\end{array}\right]$, and the first $(K+1) \times(K+1)$ block of $\mathbf{I}^{-1}$ is given by $\left(\mathbf{A}-\mathbf{B D}^{-1} \mathbf{B}^{T}\right)^{-1}$ by the block matrix inversion formula. 
From (7), (8) and (10)-(12), $\mathbf{B D}^{-1} \mathbf{B}^{T}$ can be shown to be an all-zeros matrix except for the element in the first row and first column, which is given by $\xi$ in (19). Then, the block matrix inversion formula can be applied to $\mathbf{A}-\mathbf{B D}^{-1} \mathbf{B}^{T}$ in order to obtain the CRLB as follows

$$
\left[\mathbf{I}^{-1}\right]_{11}=\left[\left(\mathbf{A}-\mathbf{B D}^{-1} \mathbf{B}^{T}\right)^{-1}\right]_{11}=\frac{1}{\left(\mathrm{I}_{\tau \tau}-\xi\right)-\mathbf{I}_{\tau a} \mathbf{I}_{a a}^{-1} \mathbf{I}_{\tau a}^{T}}
$$

From (5), (6) and (9), (33) can be shown to be equal to the CRLB expression in (18) and (19).

\section{REFERENCES}

[1] J. Mitola and G. Q. Maguire, "Cognitive radio: making software radios more personal," IEEE Personal Commun. Mag., vol. 6, no. 4, pp. 13-18, Aug. 1999.

[2] S. Haykin, "Cognitive radio: brain-empowered wireless communications," IEEE J. Select Areas Commun., vol. 23, no. 2, pp. 201-220, Feb. 2005.

[3] H. Arslan, Cognitive Radio, Software Defined Radio, and Adaptive Wireless Systems. New York: Springer, 2007.

[4] H. Celebi and H. Arslan, "Utilization of location information in cognitive wireless networks," IEEE Wireless Commun. Mag., special issue on cognitive wireless networks, vol. 14, no. 4, pp. 6-13, Aug. 2007.

[5] — "Enabling location and environment awareness in cognitive radios," Elsevier Computer Commun., special issue on advanced locationbased services), vol. 31, no. 6, pp. 1114-1125, Apr. 2008.

[6] S. Yarkan and H. Arslan, "Exploiting location awareness towards improved wireless system design in cognitive radio," IEEE Commun. Mag., vol. 46, no. 1, pp. 128-136, Jan. 2008.

[7] H. Celebi and H. Arslan, "Cognitive positioning systems," IEEE Trans. Wireless Commun., vol. 6, no. 12, pp. 4475-4483, Dec. 2007.
[8] —_, "Adaptive positioning systems for cognitive radios," in Proc. IEEE Symposium on New Frontiers in Dynamic Spectrum Access Networks (DySpan), Dublin, Ireland, Apr. 2007, pp. 78-84.

[9] J. J. Caffery, Wireless Location in CDMA Cellular Radio Systems. Boston: Kluwer Academic Publishers, 2000.

[10] Z. Sahinoglu, S. Gezici, and I. Guvenc, Ultra-Wideband Positioning Systems: Theoretical Limits, Ranging Algorithms, and Protocols. Cambridge University Press, 2008.

[11] D. B. Jourdan and N. Roy, "Optimal sensor placement for agent localization," in Proc. IEEE Position, Location, and Navigation Symp. (PLANS), San Diego, CA, Apr. 2006, pp. 128-139.

[12] J. Schroeder, S. Galler, K. Kyamakya, and K. Jobmann, "Practical considerations for optimal three dimensional localization," in Proc. IEEE Int. Conf. Multisensor Fusion and Integration for Intelligent Syst. (MFI), Heidelberg, Germany, Sept. 2006, pp. 439-443.

[13] Y. Qi, "Analysis of wireless geolocation in a non-line-of-sight environment," Ph.D. dissertation, Princeton University, Nov. 2003.

[14] H. Celebi and H. Arslan, "Ranging accuracy in dynamic spectrum access networks," IEEE Commun. Lett., vol. 11, no. 5, pp. 405-407, May 2007.

[15] R. I. C. Chiang, G. B. Rowe, and K. W. Sowerby, "A quantitative analysis of spectral occupancy measurements for cognitive radio," in Proc. IEEE IEEE Vehicular Technology Conference, Dublin, Ireland, Apr. 2007.

[16] T. A. Weiss and F. K. Jondral, "Spectrum pooling: an innovative strategy for the enhancement of spectrum efficiency," IEEE Commun. Mag., vol. 42, no. 3, pp. 8-14, Mar. 2004.

[17] S. Brandes, I. Cosovic, and M. Schnell, "Reduction of out-of-band radiation in OFDM based overlay systems," IEEE Commun. Lett., vol. 10, no. 6, pp. 420-422, June 2006.

[18] H. Mahmoud and H. Arslan, "Sidelobe suppression in OFDM-based spectrum sharing systems using adaptive symbol transition," IEEE Commun. Lett., vol. 12, no. 2, pp. 133-135, Feb. 2008.

[19] H. V. Poor, An Introduction to Signal Detection and Estimation. New York: Springer-Verlag, 1994.

[20] J. G. Proakis, Digital Communication, 4th ed. New York: McGraw-Hill, 2001. 